\title{
Phenotypic Description, Numerical Analysis, and Proposal for an Improved Taxonomy and Nomenclature of the Genus Zymomonas Kluyver and van Niel 1936
}

\author{
J. DE LEY AND J. SWINGS \\ Laboratory of Microbiology and Microbial Genetics, Faculty of Sciences, State University, K. L. \\ Ledeganckstraat 35, B-9000 Gent, Belgium
}

\begin{abstract}
One hundred thirty-eight phenotypic features were determined in 38 Zymomonas strains from diverse origin (Zairese fermenting palm saps, Mexican pulque, British spoiled beer, and sick cider). The similarity between all the strains was very high: 27 features were present, and 74 features were absent in all of them; 37 features were variable. By numerical analysis all strains formed one cluster above the simple matching coefficient of 0.88 . There were no significant differences between motile and nonmotile strains or between sucrosefermenting and non-sucrose-fermenting strains. A strain from sick cider in Bristol, United Kingdom, was a border case in its phenotypic features, deoxyribonucleic acid relatedness, and protein electropherograms. We propose: (i) to discontinue the use of the species name of Zymomonas anaerobia and its subspecies $Z$. anaerobia subsp. anaerobia and $Z$. anaerobia subsp. immobilis, and to include all of the strains in this species in Zymomonas mobilis; (ii) to consider Zymomonas congolensis as a nomen nudum and as a synonym of $Z$. mobilis. We concluded the following from our data: that $Z$. mobilis is the only species in the genus Zymomonas so far; that all the strains of our Zymomonas collection except one belong in $Z$. mobilis subsp. mobilis, the type strain of which is ATCC 10988; and that the cider sickness organisms belong in $Z$. mobilis subsp. pomaceae (Millis) comb. nov., with type strain T. H. Delft ( = strain I [Barker] $=$ ATCC 29192).
\end{abstract}

The taxonomy of the genus Zymomonas Kluyver and van Niel 1936 has not been studied extensively, the main reason being that only a few strains belonging to this genus have been known.

We collected some 40 strains, mainly from fermenting palm saps, fermenting Agave sap, deteriorated British beers, and sick cider. All of our strains, except one, had very similar deoxyribonucleic acid (DNA) base composition, genome size, and DNA relatedness (homology) (27). Close genetic relatedness suggests considerable phenotypic similarity. Indeed, the protein electropherograms of all strains, except one, were almost indistinguishably the same. The exception was the cider sickness organism, received as Zymomonas anaerobia var. pomaceae (J. Swings, K. Kersters, and J. De Ley, J. Gen. Microbiol., in press). In the present work we present the phenotypic description of 38 Zymomonas strains and the numerical analysis of 136 features.

\section{MATERIALS AND METHODS}

Bacterial strains and growth media. The strains used and information on their origin were listed in previous papers (27; Swings et al., J. Gen. Micro- biol., in press). They were maintained and grown on a standard medium containing (wt/vol) $2 \%$ glucose and $0.5 \%$ yeast extract (Difco) in distilled water.

Methods of testing. All tests were performed under the following conditions, except when otherwise stated. Inoculations of all liquid test media were made with one drop of a starter culture, $24 \mathrm{~h}$ old. The incubation temperature was $30 \mathrm{C}$. Most tests were followed for up to 3 weeks.

Morphological features. The size of 24 -h-old cells was measured on microphotographs from at least three areas in a fixed preparation, stained with $3 \%$ basic fuchsin for 1 min. Hucker Gram stain, Burdon lipid stain, Bartholomew and Mittwer stain for spores, and Anthony stain for capsules (25) were performed on cultures grown for $24 \mathrm{~h}$ in a standard medium. Intracellular glycogen was detected with Lugol $\mathrm{I}_{2}$ solution. Rosette formation and motility were determined by direct microscope observation of 12- to 24-h-old liquid cultures. Rhodes' method (10) was used for the detection of flagella.

Carbohydrates and derivatives as carbon source for growth and fermentation. Carbohydrates and derivatives as carbon source for growth and fermentation were tested in $9 \mathrm{ml}$ of a liquid medium containing $1 \%$ substrate, $0.5 \%$ yeast extract (Difco), and $0.003 \%$ bromothymol blue. Concentrated solutions of the carbohydrates were sterilized separately through membrane filters (Millipore Corp. or Seitz). 
The appearance of turbidity, the nature of the cell deposit, and formation of acid or gas were recorded.

Organic acids as carbon source for growth. Organic acids as carbon source for growth were tested as described by Lysenko (8), in $0.5 \%$ yeast extract at pH 7.0 with phenol red as indicator. The acids were Seitz-sterilized separately.

Media for the determination of growth factor requirement and for growth response to amino acids. Media were described by Van Pee et al. (32).

Synthetic minimal medium. Synthetic minimal medium was made up as described by Kluyver and Hoppenbrouwers (6). It consisted of $2 \%$ glucose, $0.1 \%$ $\mathrm{K}_{2} \mathrm{HPO}_{4}, 0.1 \%\left(\mathrm{NH}_{4}\right)_{2} \mathrm{SO}_{4}$, and $0.05 \% \mathrm{MgSO}_{4} \cdot 7 \mathrm{H}_{2} \mathrm{O}$ in water. Transfers were made with one drop.

Production of ethanol. A solution of $80 \mathrm{ml}$ of $8 \%$ glucose and $1.75 \%$ yeast extract (Difco) was inoculated with $0.5 \mathrm{ml}$ of a 24-h-old culture and incubated for 4 days at $30 \mathrm{C}$. The $\mathrm{pH}$ was brought to 10 , and the ethanol was distilled off and determined pycnometrically.

Indole formation. In $1 \%$ tryptone, $0.1 \%$ yeast extract (Difco), and 1\% glucose, indole formation was detected with the Kovacs reagent (25).

L-Lysine, L-arginine, and L-ornithine decarboxylases. L-Lysine, L-arginine, and L-ornithine decarboxylases were tested as described by Falkow (5) in $0.5 \%$ peptone, $0.3 \%$ yeast extract, $0.1 \%$ glucose, and $0.002 \%$ bromocresol purple (pH 6.75). The amino acids were added to $0.5 \%$ final.

Oxidase. Oxidase was determined by the Kovacs (7) method.

Nitrate reduction, methyl red, and Voges-Proskauer reactions. Nitrate reduction, methyl red, and Voges-Proskauer reactions were tested in the media by the method of Millis (9).

Presence of catalase. The presence of catalase was detected with $10 \% \mathrm{H}_{2} \mathrm{O}_{2}$.

Presence of amylase. The presence of amylase was tested in a medium with $0.5 \%$ yeast extract and $0.2 \%$ soluble starch.

Presence of urease. The presence of urease was tested in $9 \mathrm{ml}$ of a medium with $0.1 \%$ yeast extract, $0.1 \%$ glucose, and $0.0012 \%$ phenol red. One milliliter of $20 \%$ urea, $2 \% \mathrm{KH}_{2} \mathrm{PO}_{4}$, and $2 \% \mathrm{~K}_{2} \mathrm{HPO}_{4}$, sterilized through a membrane filter (Millipore Corp.), was added aseptically. When urease was present, a red color appeared.

Hydrolysis of Tween 60 or 80 . Hydrolysis of Tween 60 or 80 (17) was tested in the standard medium with $0.01 \% \mathrm{CaCl}_{2}$ and $2 \%$ agar. Tween was added just before the medium solidified.

Hydrolysis of gelatin. Hydrolysis of gelatin was determined in stabs containing $12 \%$ gelatin, $0.5 \%$ yeast extract (Difco), and $2 \%$ glucose.

Hydrogen sulfide formation. To test for $\mathrm{H}_{2} \mathrm{~S}$ formation, the medium used contained $0.5 \%$ peptone, $0.5 \%$ yeast extract, $2 \%$ glucose, $0.01 \%$ cystine, and $0.05 \% \mathrm{Na}_{2} \mathrm{SO}_{4}$ (pH 7). This medium was slightly adapted from one described by Skerman (19) to meet the requirements of $Z$ ymomonas. $\mathrm{H}_{2} \mathrm{~S}$ was detected with lead acetate paper strips.

Reduction of TTC. 2,3,5-Triphenyltetrazolium chloride (TTC) was sterilized cold before addition to the solid standard medium.
Reduction of dyes. Neutral red, safranine $T$, methylene blue, and thionine were tested in liquid standard media at $0.001 \%$.

pH tolerance for growth. The $\mathrm{pH}$ of the liquid standard medium was adjusted with either $\mathrm{NaOH}$ or $\mathrm{HCl}$ before sterilization; the values after sterilization are reported below.

Temperatures for growth. Temperatures for growth in liquid standard medium were tested in a water bath.

Susceptibility to high glucose concentrations. The liquid media used to test susceptibility to high glucose concentrations ( 20 and $30 \%$ ) were made up in $2 \%$ yeast extract and tyndallized.

Susceptibility to ethanol. Absolute ethanol was added to the liquid standard medium.

Susceptibility to $\mathrm{NaCl}$. The required media were made up by addition of $\mathrm{NaCl}$ to liquid standard medium before sterilization.

Susceptibility to antibiotics. Susceptibility to antibiotics was tested with 6-mm disks (Difco or Oxoid) on the inoculated surface of the solid standard medium; a second layer of the same medium was poured on to insure semi-anaerobic conditions. Readings were made after 1,2 , and 3 days. Only zones of over $2 \mathrm{~mm}$ around each disk were taken into account (31).

Susceptibility to the vibriostatic compound $0 / 129$. Susceptibility to the vibriostatic compound $0 / 129$ (2,4-diamino, 6,7-di-isopropyl pteridine) was detected as described by Rhodes (11).

Coding of the features for computation. The 138 properties tested were coded as + for their presence, as - for their absence, and as NC if no comparison was possible.

Numerical analysis. The simple matching coefficients $\left(S_{S M}\right)(26)$ were calculated. These values were clustered both by single linkage (20) and unweighted average linkage (26).

\section{RESULTS}

Phenotypical description of Zymomonas. Of 138 features examined, 101 were identical in all of our strains, 27 features were uniformly present, and 74 were lacking. They are all listed in Table 1. Thirty-seven features were present in some strains and not in others. These features, with the frequency of occurrence of positive reactions, are listed in Table 2, whereas the strains with the individual reactions for each strain are given in Table 3 . In addition to the information given in the above-mentioned tables, the following help to describe the genus.

Morphology. The cells had a wide diameter of 1 to $1.4 \mu \mathrm{m}$. The cells from some strains (17.1 and 17.3) were curved. In other strains filamentous cells up to $28 \mu \mathrm{m}$ in length or chains of cells were seen.

pH tolerance. Forty-four percent of the strains still grew at $\mathrm{pH}$ 3.5. This high acid tolerance allowed many strains to live in acid palm wines, which mostly have a pH of 3 to 4 . The $\mathrm{pH}$ range for growth was 3.5 to 7.5 . 
TABLE 1. Features common to all Zymomonas strains tested ${ }^{a}$

\section{Features present or positive}

Rods, usually in pairs

If motile, 1-4 polar flagella

Width of cell: 1-1.2 $\mu \mathrm{m}$

Length of cell: $2-6 \mu \mathrm{m}$

Colonies in standard medium: white or cream-colored, glistening

Colony diam in standard medium: 1-2 mm after 2 days

Regular colony edge

Fruity odor

Growth in $2 \%$ yeast extract $+20 \%$ glucose

Growth in solid standard medium $+0.1 \%$ neutral red

Growth on and fermentation of fructose and glucose

Formation of traces of acetylmethylcarbinol

Presence of catalase
Reduction of methylene blue

Reduction of thionin

Reduction of 2,3,5-triphenyltetrazolium chloride

Final $\mathrm{pH}$ in liquid standard medium ( 3 days, $30 \mathrm{C}$ ): 4.8 5.2

Optimal pH for growth: 7.3

Growth in liquid standard medium $+5 \%$ ethanol

Ratio $\frac{\text { mol ethanol produced }}{\text { mol glucose fermented }}>1.5$

Gas $\left(\mathrm{CO}_{2}\right)$ production from glucose and fructose

Susceptible to $10 \mu \mathrm{g}$ of tetracycline

Susceptible to $10 \mu \mathrm{g}$ of fusidic acid

Susceptible to $500 \mu \mathrm{g}$ of sulfafurazole

Susceptible to $30 \mu \mathrm{g}$ of novobiocin

Requirement for pantothenate ${ }^{b}$

Requirement for biotin ${ }^{b}$

Features absent or negative

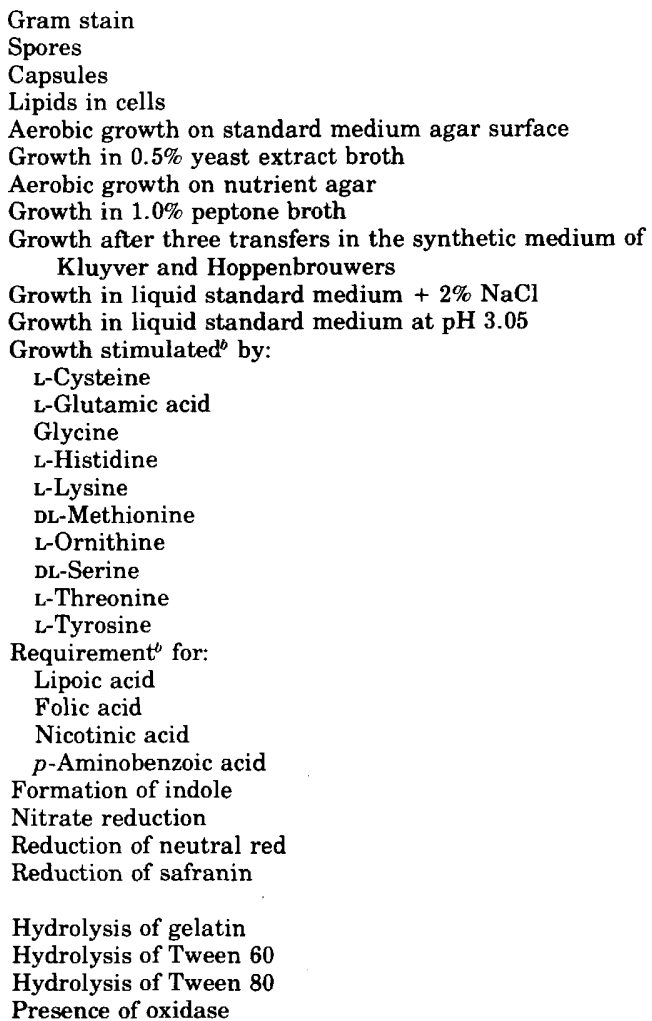

\section{Growth on: \\ Starch \\ Dextrin \\ Raffinose \\ D-Trehalose \\ Maltose \\ Lactose \\ D-Cellobiose \\ D-Galactose \\ D-Mannose \\ L-Sorbose \\ Salicin \\ L-Rhamnose \\ D-Arabinose \\ L-Arabinose \\ D-Xylose \\ D-Ribose \\ D-Sorbitol \\ Dulcitol \\ D-Mannitol \\ Adonitol \\ Erythritol \\ Glycerol}

Ethanol

Sodium D-galacturonate

Sodium D,L-malate

Sodium succinate

Sodium pyruvate

Sodium D,L-lactate

Sodium tartrate

Sodium citrate

Susceptibility ${ }^{b}$ to:

$0.01 \%$ actidione

$5 \mathrm{U}$ of bacitracin

$10 \mu \mathrm{g}$ of gentamicin

$10 \mu \mathrm{g}$ of kanamycin

$10 \mu \mathrm{g}$ of lincomycin

$30 \mu \mathrm{g}$ of nalidixic acid

$10 \mu \mathrm{g}$ of neomycin

$5 \mathrm{U}$ of penicillin

$300 \mathrm{U}$ of polymyxin

$10 \mu \mathrm{g}$ of streptomycin

Vibriostatic compound $0 / 129$

\footnotetext{
"Standard medium contains $2 \%$ glucose $+0.5 \%$ yeast extract.
}

${ }^{b}$ Data from Van Pee et al. $(31,32)$. 
TABLE 2. Differential features ${ }^{a}$

\begin{tabular}{|c|c|}
\hline Feature & $\begin{array}{l}\text { No. of positive } \\
\text { strains }\end{array}$ \\
\hline 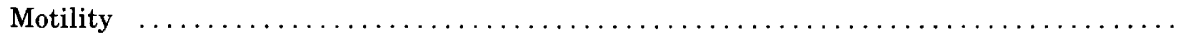 & 10 \\
\hline Formation of rosettes $\ldots \ldots \ldots \ldots \ldots \ldots \ldots \ldots \ldots \ldots \ldots \ldots \ldots \ldots \ldots \ldots$ & 19 \\
\hline 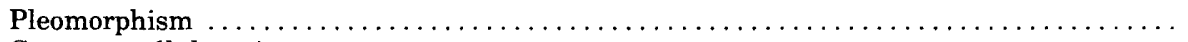 & 24 \\
\hline Compact cell deposit $\ldots \ldots \ldots \ldots \ldots \ldots \ldots \ldots \ldots \ldots \ldots \ldots \ldots \ldots \ldots \ldots \ldots \ldots$ & 25 \\
\hline 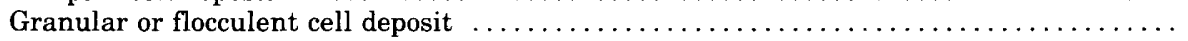 & 13 \\
\hline Growth on and fermentation of sucrose $\quad \ldots \ldots \ldots \ldots \ldots \ldots \ldots \ldots \ldots \ldots \ldots \ldots \ldots$ & 19 \\
\hline Formation of $\mathrm{H}_{2} \mathrm{~S}$ & 4 \\
\hline 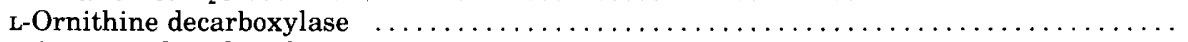 & 4 \\
\hline L-Arginine decarboxylase $\ldots \ldots \ldots$ & 31 \\
\hline L-Lysine decarboxylase $\ldots \ldots \ldots$ & 18 \\
\hline 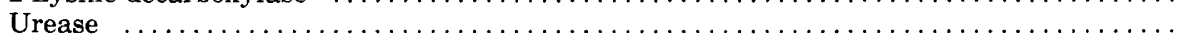 & 9 \\
\hline 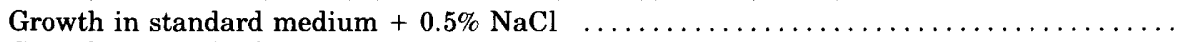 & 37 \\
\hline 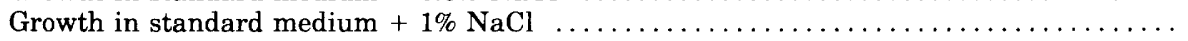 & 27 \\
\hline 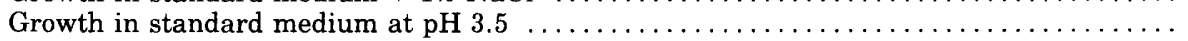 & 17 \\
\hline 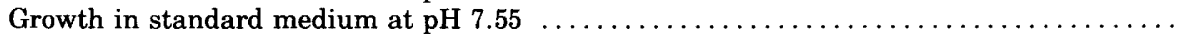 & 32 \\
\hline 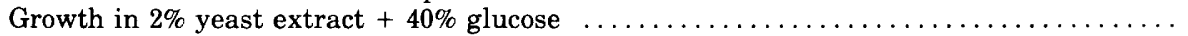 & 21 \\
\hline 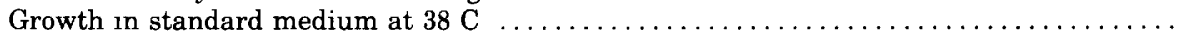 & 28 \\
\hline 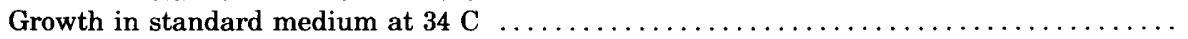 & 37 \\
\hline Final $\mathrm{pH}$ after growth in standard medium at $34 \mathrm{C}$, for 7 days: $<4.8 \ldots \ldots \ldots \ldots$ & 1 \\
\hline Growth after a second transfer in the synthetic medium of Kluyver and Hoppenbrouwers . & 4 \\
\hline \multicolumn{2}{|l|}{ Growth stimulated ${ }^{b}$ by: } \\
\hline 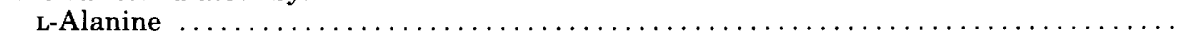 & 3 \\
\hline 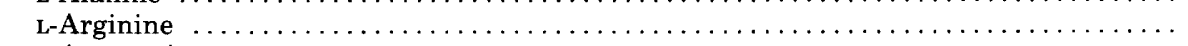 & 4 \\
\hline L-Asparagine & 2 \\
\hline 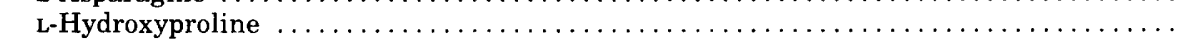 & 4 \\
\hline 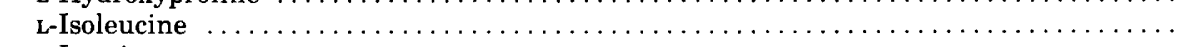 & 2 \\
\hline 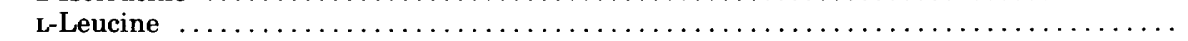 & 8 \\
\hline 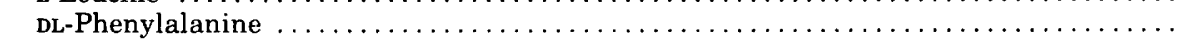 & 1 \\
\hline 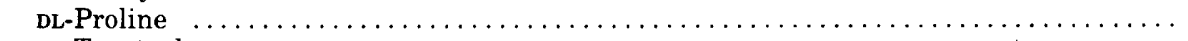 & 2 \\
\hline 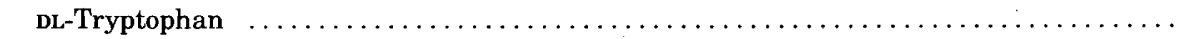 & 1 \\
\hline 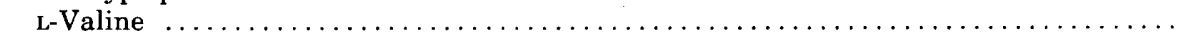 & 18 \\
\hline Riboflavin requirement $t^{b}$ & 3 \\
\hline Thiamin requirement $t^{b} \ldots \ldots \ldots \ldots$ & 1 \\
\hline Cyanocobalamine requirement & 3 \\
\hline \multicolumn{2}{|l|}{ Resistance to: $^{b}$} \\
\hline Ampicillin, $10 \mu \mathrm{g}$ & 15 \\
\hline Cephaloridine, $10 \mu \mathrm{g} \quad \ldots$. & 11 \\
\hline 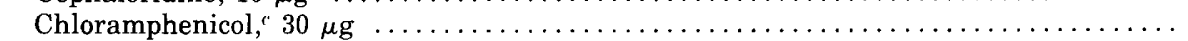 & 1 \\
\hline 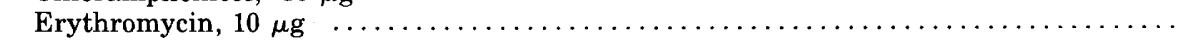 & 34 \\
\hline 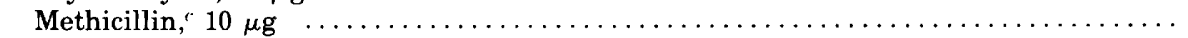 & 37 \\
\hline 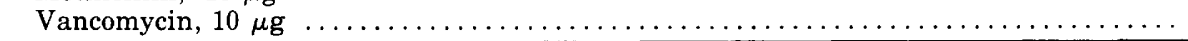 & 20 \\
\hline
\end{tabular}

${ }^{a}$ The total number of strains used was 38 . Standard medium contains $0.5 \%$ yeast extract and $2 \%$ glucose.

${ }^{b}$ Data from Van Pee et al. $(31,32)$.

" Data not used for $\mathrm{S}_{\mathrm{SM}}$ calculation.

Sensitivity to ethanol. All strains grew readily in the presence of $5 \%$ ethanol. Many strains still grew in $10 \%$ ethanol. In most palm wines the ethanol content is 3 to $5 \%$.

Effect of high glucose concentrations. Kluyver and Hoppenbrouwers (6) reported that their strain (ATCC 10988) of $Z$. mobilis grew with fermentation in a medium containing $25 \%$ glucose. All of our strains grew on a $20 \%$ glucose medium. Only strains VP1 and VP3 did not grow on a $30 \%$ glucose medium.

Growth on and fermentation of sucrose. Some authors considered the features of growth on and fermentation of sucrose to be the key differences between Zymomonas mobilis and $Z$. anaerobia (see below). In our collection, 17 Zairese palm-wine Zymomonas strains and the named $Z$. mobilis strain 10988 (and its subculture 410) grew on and fermented sucrose. Sixteen other Zairese palm-wine Zymomonas strains and three named $Z$. anaerobia strains neither grew on nor fermented this substrate. For the latter strains this was not surprising as they had been named $Z$. anaerobia because they did not ferment sucrose.

Formation of acetylmethylcarbinol. We found traces of acetylmethylcarbinol formation with all of our strains. This extends the 


\begin{tabular}{|c|c|c|c|c|c|c|c|c|c|c|c|c|c|c|c|c|c|c|c|c|}
\hline 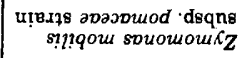 & Z6I6Z OOLU & 1 & 1 & 1 & + & 1 & + & 1 & 1 & 1 & 1 & + & 1 & 1 & 1 & 1 & + & 1 & + & \\
\hline \multirow{37}{*}{ 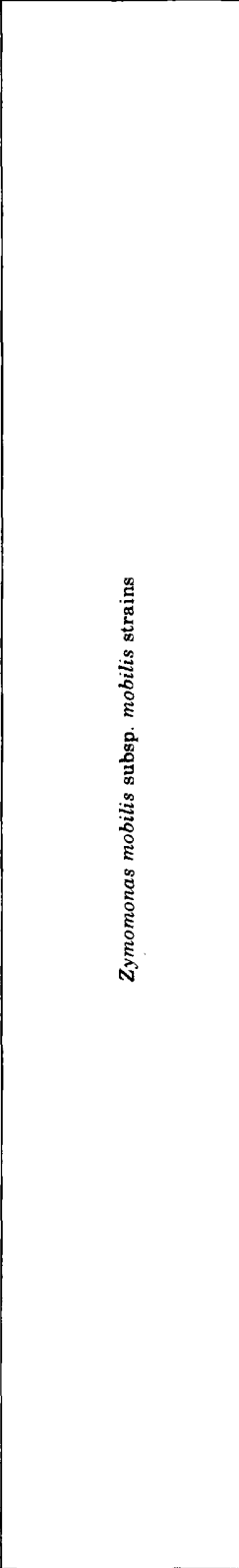 } & 8860! OOLV & + & 1 & 1 & + & 1 & 1 & + & + & + & 1 & + & + & + & + & 1 & + & + & 1 & \\
\hline & 60† рив;suarno & + & 1 & + & + & 1 & + & 1 & + & + & 1 & 1 & 1 & I & + & 1 & 1 & 1 & 1 & 1 \\
\hline & 0It pur[suarne & + & 1 & 1 & + & 1 & + & + & + & + & 1 & + & + & + & + & 1 & + & + & 1 & 1 \\
\hline & $\angle Z Z 8$ gIDN & + & + & + & + & 1 & 1 & 1 & + & + & 1 & 1 & 1 & 1 & + & 1 & + & + & 1 & I \\
\hline & +2 & 1 & + & t & + & 1 & + & 1 & + & 1 & + & + & 1 & + & + & 1 & + & + & 1 & + \\
\hline & $\mathrm{Fd} \Lambda$ & 1 & + & + & 1 & + & 1 & 1 & + & 1 & + & 1 & + & 1 & + & 1 & 1 & 1 & 1 & 1 \\
\hline & $8 \mathrm{~d} \Lambda$ & 1 & + & + & 1 & + & 1 & 1 & + & 1 & + & + & 1 & + & + & 1 & + & 1 & 1 & 1 \\
\hline & $\mathrm{Zd} \Lambda$ & 1 & + & + & + & 1 & 1 & 1 & + & 1 & + & 1 & + & + & + & 1 & + & 1 & 1 & + \\
\hline & $\operatorname{Id} \Lambda$ & 1 & + & t & + & 1 & 1 & 1 & + & 1 & + & + & 1 & + & + & 1 & 1 & 1 & 1 & 1 \\
\hline & $8 \mathrm{Z}$ & 1 & + & + & + & 1 & + & + & + & + & + & + & + & + & + & + & + & + & 1 & 1 \\
\hline & $L Z$ & 1 & + & 1 & + & 1 & + & 1 & + & + & + & + & + & + & + & 1 & + & 1 & 1 & 1 \\
\hline & โ $616 Z \mathrm{DOL} \mathrm{H}=9 \mathrm{Z}$ & 1 & 1 & 1 & + & 1 & + & + & + & + & + & + & + & + & + & 1 & 1 & 1 & 1 & 1 \\
\hline & $\Phi \mathbf{Z}$ & 1 & I & 1 & + & 1 & 1 & + & + & + & + & + & + & $1+$ & + & 1 & + & 1 & 1 & 1 \\
\hline & $\varepsilon z$ & 1 & + & 1 & + & 1 & + & + & + & + & + & + & + & + & + & 1 & + & 1 & 1 & 1 \\
\hline & $\mathrm{ZZ}$ & 1 & 1 & 1 & + & 1 & + & 1 & + & + & + & + & + & + & + & 1 & + & 1 & 1 & 1 \\
\hline & $\mathrm{Iz}$ & 1 & + & + & + & 1 & + & + & + & + & + & + & + & + & + & 1 & + & 1 & 1 & 1 \\
\hline & $\$ 0 L$ & 1 & + & 1 & 1 & + & + & + & + & 1 & 1 & + & 1 & + & + & 1 & + & 1 & 1 & + \\
\hline & $8 \mathrm{I} \cdot 0 \mathrm{~L}$ & 1 & 1 & 1 & + & 1 & + & 1 & + & 1 & 1 & + & + & + & + & 1 & + & + & 1 & 1 \\
\hline & It 02 & 1 & 1 & 1 & + & 1 & 1 & 1 & + & + & 1 & I & + & + & + & 1 & + & + & 1 & 1 \\
\hline & $0 \mathrm{I}^{\circ} \mathrm{OL}$ & 1 & 1 & + & + & 1 & 1 & 1 & + & 1 & + & + & + & + & + & 1 & + & + & 1 & 1 \\
\hline & 6.02 & 1 & 1 & 1 & + & 1 & + & 1 & + & + & 1 & + & + & + & + & 1 & + & + & 1 & 1 \\
\hline & $\angle .02$ & 1 & + & 1 & 1 & + & + & 1 & + & 1 & I & + & + & 1 & + & + & 1 & 1 & 1 & + \\
\hline & $80 L$ & 1 & 1 & 1 & + & 1 & + & + & + & + & 1 & + & + & + & + & 1 & + & + & 1 & I \\
\hline & $Z^{\prime} 02$ & 1 & + & 1 & 1 & + & + & 1 & + & + & + & + & + & 1 & + & 1 & + & + & 1 & + \\
\hline & {$[0 L$} & 1 & 1 & 1. & + & 1 & + & 1 & + & 1 & 1 & + & + & + & + & 1 & + & + & 1 & 1 \\
\hline & 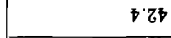 & 1 & + & 1 & 1 & + & + & + & + & + & 1 & + & 1 & + & + & 1 & + & 1 & 1 & 1 \\
\hline & $87 t$ & 1 & + & 1 & 1 & + & 1 & + & + & + & 1 & + & 1 & + & + & 1 & + & 1 & 1 & 1 \\
\hline & 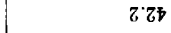 & 1 & 1 & 1 & 1 & + & + & + & + & + & 1 & + & 1 & + & + & 1 & + & 1 & 1 & 1 \\
\hline & I $z \triangleright$ & 1 & 1 & 1 & 1 & + & 1 & + & + & + & 1 & + & 1 & + & + & 1 & + & 1 & 1 & 1 \\
\hline & $\nabla .2 \mathrm{I}$ & 1 & + & 1 & 1 & + & + & 1 & + & + & 1 & + & 1 & 1 & + & + & + & + & 1 & + \\
\hline & $\varepsilon \angle \mathrm{I}$ & 1 & 1 & 1 & 1 & + & + & + & + & + & 1 & + & + & 1 & + & 1 & + & 1 & 1 & + \\
\hline & $z<\mathrm{t}$ & 1 & + & 1 & 1 & + & + & 1 & + & $t$ & + & + & 1 & 1 & + & + & + & + & 1 & + \\
\hline & 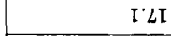 & 1 & + & 1 & 1 & + & + & + & + & + & 1 & + & + & 1 & + & 1 & + & + & 1 & + \\
\hline & $g^{\prime} s$ & 1 & 1 & 1 & + & 1 & + & + & + & + & 1 & + & 1 & \pm & + & 1 & + & + & 1 & 1 \\
\hline & $\nabla \cdot \mathbf{s}$ & 1 & 1 & 1 & + & 1 & 1 & + & + & + & 1 & + & 1 & + & + & 1 & + & + & 1 & 1 \\
\hline & 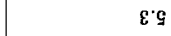 & 1 & 1 & 1 & + & 1 & 1 & + & + & + & + & + & 1 & + & + & 1 & 1 & + & 1 & 1 \\
\hline & I'G & 1 & 1 & । & + & 1 & 1 & + & + & + & + & + & 1 & + & + & 1 & 1 & 1 & 1 & 1 \\
\hline \multicolumn{2}{|l|}{ 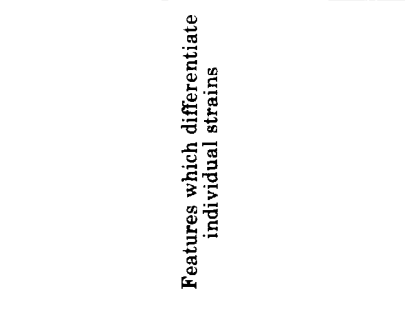 } & 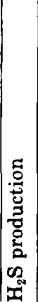 & 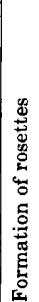 & : & 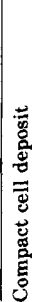 & 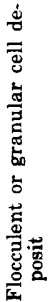 & 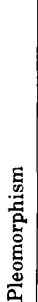 & 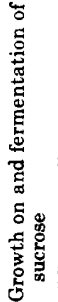 & 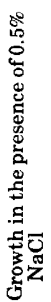 & 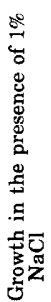 & 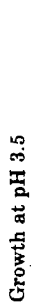 & 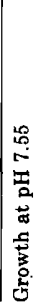 & 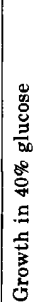 & 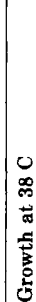 & 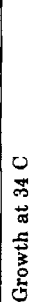 & 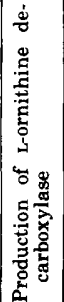 & 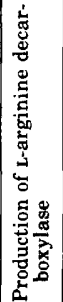 & 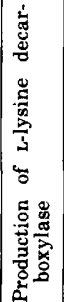 & 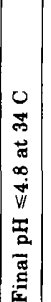 & 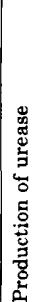 \\
\hline
\end{tabular}




\begin{tabular}{|c|c|c|c|c|c|c|c|c|c|c|c|c|c|c|c|c|c|}
\hline 1 & + & 1 & 1 & 1 & + & + & + & + & 1 & 1 & 1 & 1 & 1 & + & 1 & + & 1 \\
\hline+ & 1 & + & 1 & + & 1 & 1 & + & 1 & 1 & 1 & 1 & 1 & 1 & 1 & 1 & 1 & 1 \\
\hline+ & + & + & + & + & 1 & + & + & + & + & 1 & 1 & 1 & 1 & 1 & 1 & 1 & 1 \\
\hline+ & + & + & + & + & 1 & 1 & + & 1 & 1 & 1 & 1 & 1 & 1 & 1 & 1 & 1 & 1 \\
\hline+ & + & + & + & + & 1 & 1 & + & + & + & + & 1 & 1 & 1 & 1 & 1 & 1 & 1 \\
\hline 1 & + & 1 & + & 1 & + & 1 & + & 1 & 1 & 1 & 1 & 1 & 1 & 1 & 1 & 1 & 1 \\
\hline 1 & 1 & 1 & + & 1 & 1 & $i$ & 1 & 1 & 1 & 1 & 1 & 1 & 1 & 1 & 1 & 1 & 1 \\
\hline+ & 1 & 1 & + & 1 & + & 1. & + & 1 & + & 1 & 1 & 1 & 1 & 1 & 1 & 1 & + \\
\hline+ & + & + & + & 1 & 1 & 1 & + & 1 & 1 & 1 & 1 & 1 & 1 & 1 & 1 & 1 & 1 \\
\hline+ & + & + & + & 1 & z & $\mathbf{z}$ & $\mathbf{z}$ & Z & 0 & $\mathbf{z}$ & $\mathbf{z}$ & $\mathbf{z}$ & $\mathbf{z}$ & z & $\mathbf{z}$ & $\begin{array}{l}0 \\
\mathbf{z}\end{array}$ & z \\
\hline 1 & + & 1 & + & 1 & 1 & 1 & 1 & 1 & 1 & 1 & 1 & 1 & 1 & 1 & + & 1 & 1 \\
\hline+ & + & 1 & + & 1 & 1 & 1 & 1 & 1 & 1 & + & 1 & 1 & 1 & 1 & + & 1 & 1 \\
\hline+ & + & 1 & + & 1 & 1 & 1 & 1 & 1 & 1 & 1 & 1 & 1 & 1 & 1 & 1 & 1 & 1 \\
\hline 1 & 1 & 1 & + & 1 & 1 & 1 & + & 1 & 1 & 1 & 1 & 1 & 1 & 1 & 1 & 1 & 1 \\
\hline+ & 1 & 1 & + & 1 & 1 & 1 & 1 & 1 & 1 & 1 & 1 & 1 & 1 & 1 & 1 & 1 & 1 \\
\hline 1 & + & 1 & + & 1 & 1 & 1 & 1 & 1 & 1 & 1 & 1 & 1 & 1 & 1 & 1 & 1 & 1 \\
\hline+ & 1 & 1 & + & 1 & 1 & 1 & 1 & 1 & 1 & 1 & 1 & 1 & 1 & 1 & 1 & 1 & 1 \\
\hline 1 & 1 & 1 & + & 1 & 1 & 1 & + & 1 & + & 1 & 1 & 1 & 1 & 1 & 1 & 1 & 1 \\
\hline+ & + & + & + & 1 & 1 & 1 & 1 & 1 & 1 & 1 & 1 & 1 & 1 & 1 & 1 & 1 & 1 \\
\hline 1 & 1 & 1 & + & 1 & z & z & $\dot{z}$ & $z$ & $\mathbf{z}$ & Z & 0 & $\begin{array}{l}0 \\
z\end{array}$ & z & z & z & $\tilde{z}$ & $\tilde{z}$ \\
\hline 1 & 1 & + & + & 1 & 1 & 1 & + & + & + & 1 & 1 & 1 & 1 & 1 & 1 & 1 & + \\
\hline+ & + & 1 & + & 1 & 1 & 1 & + & 1 & 1 & 1 & 1 & 1 & 1 & 1 & 1 & 1 & + \\
\hline 1 & 1 & 1 & 1 & 1 & 0 & $\begin{array}{l}0 \\
z\end{array}$ & $z$ & 0 & 0 & $\begin{array}{l}0 \\
\mathbf{z}\end{array}$ & z & 0 & $\mathbf{z}$ & z & z & Z & $z$ \\
\hline+ & + & + & + & 1 & 1 & 1 & + & 1 & 1 & 1 & 1 & 1 & 1 & 1 & 1 & 1 & 1 \\
\hline 1 & + & 1 & + & 1 & 1 & 1 & + & 1 & + & 1 & + & 1 & 1 & 1 & 1 & 1 & 1 \\
\hline+ & + & + & + & 1 & 1 & 1 & 1 & 1 & 1 & 1 & 1 & 1 & 1 & 1 & 1 & 1 & 1 \\
\hline 1 & 1 & 1 & + & 1 & 1 & 1 & 1 & 1 & + & 1 & + & 1 & 1 & 1 & 1 & 1 & 1 \\
\hline 1 & + & 1 & + & 1 & 1 & 1 & 1 & 1 & 1 & 1 & 1 & 1 & + & 1 & 1 & 1 & 1 \\
\hline 1 & 1 & 1 & + & 1 & 1 & 1 & 1 & 1 & 1 & 1 & 1 & 1 & 1 & 1 & 1 & 1 & 1 \\
\hline 1 & 1 & 1 & + & 1 & 1 & 1 & + & 1 & 1 & 1 & 1 & 1 & 1 & 1 & 1 & 1 & 1 \\
\hline 1 & 1 & + & + & 1 & 1 & 1 & 1 & 1 & 1 & 1 & 1 & 1 & + & 1 & 1 & 1 & 1 \\
\hline 1 & 1 & 1 & 1 & 1 & 1 & 1 & 1 & 1 & 1 & + & 1 & + & 1 & 1 & 1 & 1 & 1 \\
\hline 1 & 1 & 1 & + & 1 & 1 & 1 & + & 1 & 1 & 1 & 1 & 1 & 1 & 1 & 1 & 1 & 1 \\
\hline 1 & 1 & 1 & + & 1 & 1 & 1 & + & 1 & 1 & 1 & 1 & 1 & 1 & 1 & 1 & 1 & 1 \\
\hline 1 & + & + & + & 1 & 1 & 1 & + & 1 & + & 1 & 1 & + & 1 & 1 & 1 & 1 & 1 \\
\hline 1 & + & 1 & + & 1 & 1 & 1 & 1 & 1 & 1 & + & + & 1 & 1 & 1 & 1 & 1 & 1 \\
\hline 1 & 1 & 1 & + & 1 & 1 & 1 & 1 & 1 & 1 & 1 & 1 & 1 & 1 & 1 & 1 & 1 & 1 \\
\hline 1 & + & 1 & + & 1 & $i$ & 1 & 1 & 1 & 1 & 1 & 1 & 1 & $!$ & 1 & 1 & 1 & 1 \\
\hline 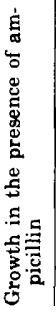 & 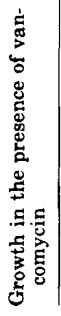 & 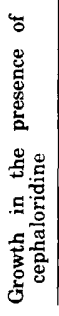 & 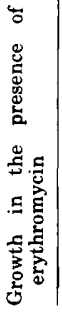 & 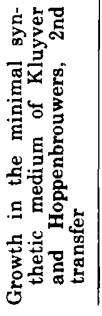 & 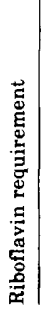 & 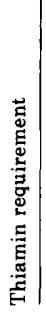 & 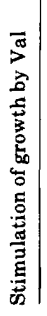 & 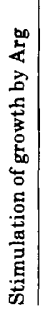 & 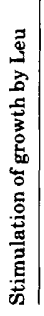 & 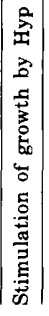 & 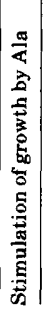 & 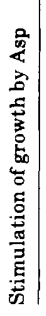 & 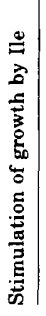 & 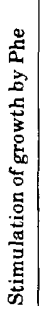 & 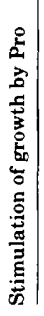 & 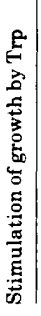 & 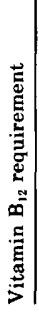 \\
\hline
\end{tabular}


findings by Kluyver and Hoppenbrouwers (6), Tankó (28), and Schreder et al. (14). Shimwell (15) and Millis (9) found no reaction.

Reproducibility. Two subcultures from our strain of $Z$. anaerobia subsp. pomaceae were submitted separately to the battery of tests. Five tests of 138 (3.6\%) did not give reproducible results. $Z$. mobilis strain ATCC 10988 was compared with Queensland 410 , and $Z$. anaerobia NCIB 8227 was compared with Queensland 409. The Queensland strains are subcultures from the respective ATCC and NCIB parent cultures. In both cases each pair of strains displayed six non-reproducible tests $(4.4 \%)$. Our findings are in excellent agreement with those of Sneath and Johnson (22) and Snell and Lapage (24), who reported 2.2 and $5 \%$ non-reproducible tests, respectively. In spite of very careful experimenting and repeating of our tests, a few tests remain non-reproducible; this is statistically unavoidable (21).

Numerical analysis. The results of the unweighted average linkage are represented in Fig. 1. The single-linkage dendrogram (not shown here) was very similar. All strains formed a dense cluster with at least $88 \% \mathrm{~S}_{\mathrm{SM}}$ in the first type of calculation (Fig. 1) and $90 \% \mathrm{~S}_{\mathrm{SM}}$ in the second procedure, reflecting a high phenotypic relatedness. The bulk of the group was made up of the Zairese palm-wine strains. In view of the reproducibility limits, all strains above the $95 \% \mathrm{~S}_{\mathrm{SM}}$ link must be regarded as taxonomically identical. Whether these strains were isolated from Elaeis sap or Raphia sap either in the western tip or in the northwest part of Zaire, they were all the same, except for a few (less than 12) individual differences.

Both subcultures of the named $Z$. mobilis strain from Mexican Agave sap were phenotypically almost the same as the Zairese strains; in particular they were indistinguishable from strains isolated from fermenting Elaeis sap around Kinshasa, Zaïre, in 1970 by one of us (J.S.).

The named strain $Z$. anaerobia NCIB 8227 and its subculture Queensland 409 were just at the border of this main group with an average of 15 different features. However, there was no single phenotypic property which consistently differentiated both strains from all of the others. The average phenotypical similarity of both strains with the main group of our strains was as high as two Zairese strains, 17.3 and 70.7 .

The cider organism formed the border of the group, with an average difference of 17 features. However, phenotypically there were only five minor properties consistently differentiat- ing this strain from all of the others (Table 4).

Phenotypic centrotype. We calculated the phenotypic homogeneity of the cluster and the most representative strain by a slight modification of the methods of Rogers and Tanimoto (13) and of Silvestri et al. (18). We defined the value of the ranking order $R$ as the number of strains with which the strain under consideration differs with less than 10 features. The hierarchical order $\mathrm{H}$ of each strain was defined here as the sum of its identical features with every other strain minus 3,700 . The results are shown in Fig. 2. All of the strains formed a homogeneous cloud, except five border cases. The most typical strains were those from the $\mathrm{Z}$ and the 70 series from fermenting Elaeis sap in the neighborhood of Kinshasa, Zaire. Because it had the highest $R$ value, strain $Z 6$ was the phenotypic centrotype. It was also the centroid strain with the most typical electropherogram (Swings et al., J. Gen. Microbiol., in press) and the most representative strain of $Z$. mobilis subsp. mobilis. It was deposited as NCIB 11199 and ATCC 29191.

\section{DISCUSSION}

We shall discuss here the taxonomic implications from the present phenotypic analysis as well as from our previous results on protein electropherograms (Swings et al., J. Gen. Microbiol., in press) and DNA properties (27). All of our strains, except the sick cider strain, formed one tight group with $48.5 \pm 1.0 \mathrm{~mol} \%$ guanine plus cytosine $(\mathrm{G}+\mathrm{C})$, over $76 \% \mathrm{D}$ (degree of DNA relatedness in stringent conditions), a genome molecular weight of $1.5 \times 10^{9}$, almost identical protein electropherograms, and over $88 \%$ of phenotypic similarity $\mathrm{S}_{\mathrm{SM}}$. This group contains 21 Zymomonas strains isolated from fermenting Elaeis sap in western Zaïre, twelve strains from fermenting Raphia sap in the northwest part of Zaïre, two subcultures of the type strain of $Z$. mobilis, ATCC 10988, isolated from Agave sap in Mexico, two strains from Arenga sap in Indonesia, one named $Z$. mobilis strain from British ale, and four cultures named $Z$. anaerobia from spoiled beer in Britain. All of these strains are so similar that they deserve to be united in one taxon, which we propose to call Zymomonas mobilis subsp. mobilis. Sneath and Skerman (23) proposed strain ATCC 10988 as cotype for $Z$. mobilis. We propose it as the lectotype strain for $Z$. mobilis subsp. mobilis. Strain ATCC $10988=$ NCIB $8938=$ NRRL B-806 $=$ L192 (Lab. Microbiol., Techn. Univ., Delft, the Netherlands) $=$ strain 1 (van der Toorn) = Ampoule $\mathrm{n}^{\circ} 410$ (Dept. Microbiol., Fac. Med., Univ. 
AVERAGE NUMBER OF

DIFFERENT FEATURES
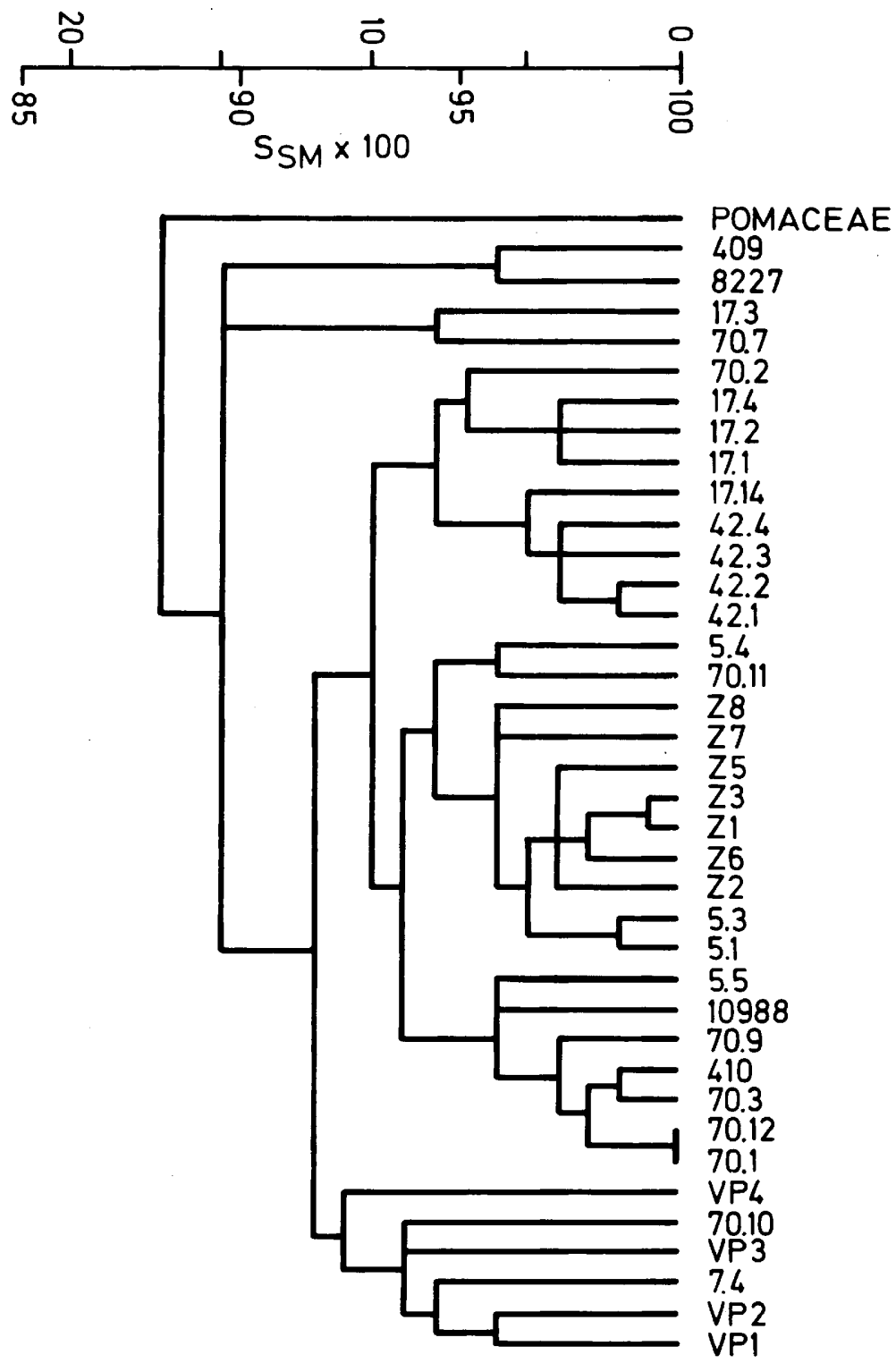

FIG. 1. Dendrogram of the $S_{\mathrm{SM}}$ similarity coefficient $\times 100$, grouped by the unweighted average-linkage cluster analysis, showing the relationships among various Zymomonas strains. Abbreviations: POMACEAE, $Z$. mobilis subsp. pomaceae ATCC 29192; 409, Z. mobilis subsp. mobilis Queensland 409 ex NCIB 8227; 8227, Z. mobilis subsp. mobilis NCIB 8227; 10988, Z. mobilis subsp. mobilis ATCC 10988; 410, Z. mobilis subsp. mobilis Queensland 410 ex NCIB 8938 ex ATCC 10988. The remaining symbols refer to strains of $Z$. mobilis subsp. mobilis isolated in Zä̈re (27; Swings et al., J. Gen. Microbiol., in press). The name Z. congolensis (29) was proposed without mention of actual strain numbers. We know that the strains Z1,Z2,Z3, $Z 5, Z 6, Z 7, Z 8, V P 1, V P 2, V P 3, V P 4$, and 7.4 (30) were concerned.

Queensland, Australia) = DSM $424=$ IMG 1655. This strain was isolated by P. Lindner from pulque from Agave americana in 1924 in
Mexico and sent by him in 1929 to Ir. A. J. Kluyver, Lab. Microbiology, Technical University, Delft, The Netherlands. From there it was 
TABLE 4. Differential features of the cider sickness organism Z. mobilis subsp. pomaceae ATCC 29192

Features Z. mobilis subsp. mobilis $\quad$ Z. mobilis subsp. pomaceae

Growth in standard medium + $0.5 \% \mathrm{NaCl}$

Growth in standard medium at $36 \mathrm{C}$

Growth in standard medium + $0.0075 \% \mathrm{KCN}$

Final pH after 7 days of growth at $34 \mathrm{C}$ in standard medium

Number of amino acids stimulating growth
Growth after 1-3 days (37 No growth

$$
\text { strains) }
$$

Growth after 1 day (37 strains) Scant growth after 2 days

Growth after $\mathbf{1 - 5}$ days (16

No growth

strains)

4.9-5.4 (37 strains)

5.7

0-5 (37 strains; exact number de- 6 (Arg, Gly, Phe, Thr, Trp, Val) pends on strain)

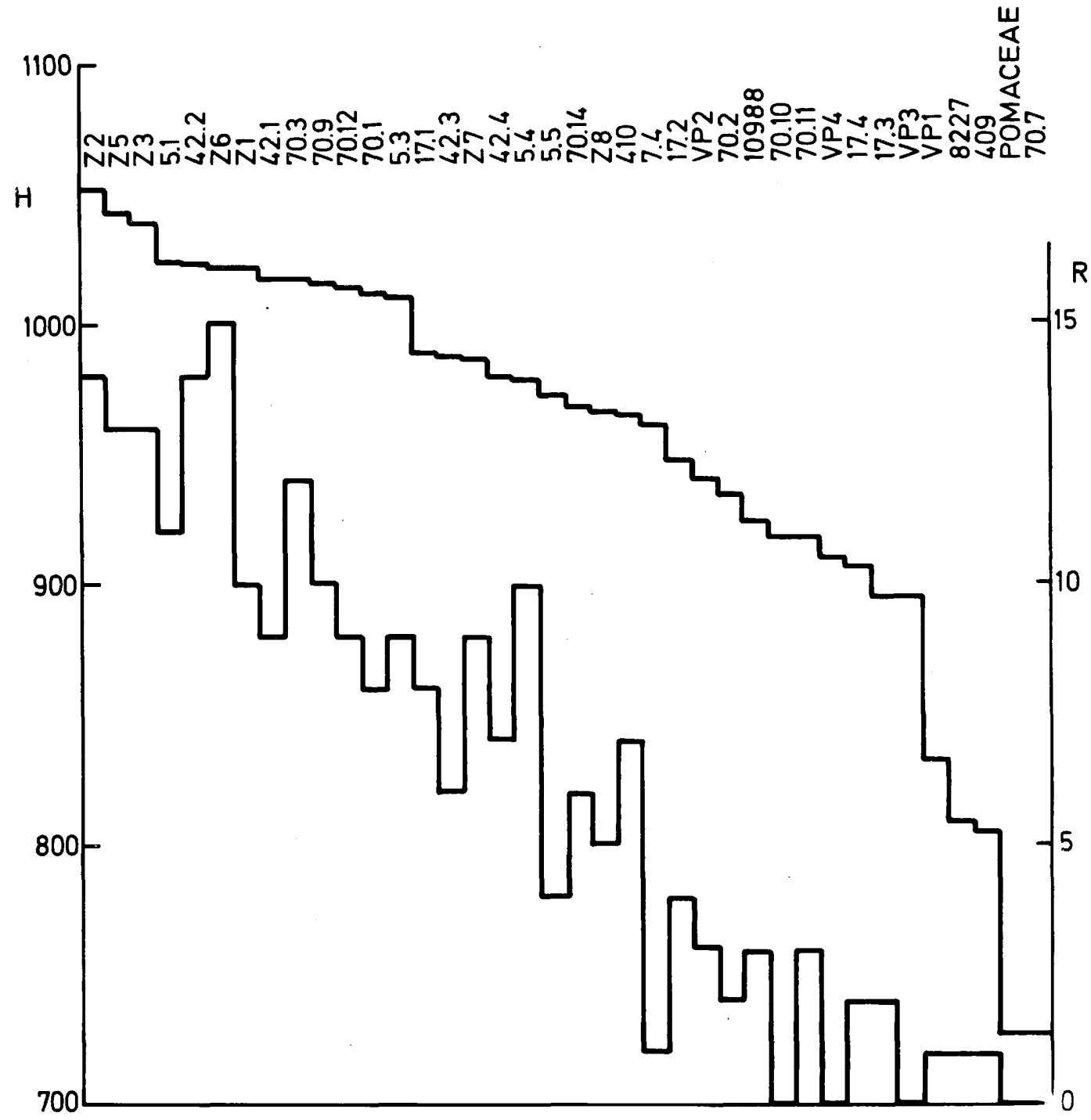

FIG. 2. Phenotypic homogeneity of Zymomonas and phenotypic centrotype Z6. The ranking order $R$ and the hierarchical order $H$ are defined in the text. 
sent to several collections and individuals.

Van Pee and Swings (29) suggested the name Zymomonas congolensis for a few strains from Zaïre. It was published as a nomen nudum; no type strain was proposed. These strains (see Fig. 1), included in the present study, are indistinguishable from our other strains. A separate species status is not justified; the name of this taxon is a subjective synonym of $Z$. mobilis. We propose to reject the name $Z$. congolensis for the above-mentioned reasons.

It was suggested (1) that the fermentation of sucrose is the main feature distinguishing $Z$. anaerobia from $Z$. mobilis. However, this conclusion was derived from work with a few strains only. The sucrose fermentation in a Zymomonas strain seems to be an inducible property. Kluyver and Hoppenbrouwers (6) observed that their strain of $Z$. mobilis lost the ability to ferment sucrose by growing it on glucose and gained it again in the presence of sucrose. An opposite phenomenon was observed by Dadds et al. (2) and by Richards and Corbey (12): subculturing in sucrose or fructose media converted sucrose non-fermenting strains into sucrose fermenters. We found no correlation whatsoever between growth on and fermentation of sucrose and any other feature from $Z y m$ omonas strains. Whether strains grow on and ferment sucrose or not, their genotypical (27) and phenotypical (Swings et al., J. Gen. Microbiol., in press; and this paper) features are almost indistinguishable. The only quantitative information on the mechanism of sucrose breakdown was given by Dawes et al. (3) with $Z$. mobilis NCIB 8938 . About $10 \%$ of the sucrose is converted to levan and glucose by a levansucrase. About $90 \%$ of the sucrose is hydrolyzed to glucose and fructose; whether this is effected by levansucrase or by an independent invertase is not known. The enzymatic difference between sucrose-fermenting and non-sucrose-fermenting zymomonads is probably one or at most two enzymes (levansucrase and/or invertase). It is not justified to create or to maintain a separate species because of such a small difference. We examined three cultures (plus two subcultures) of named $Z$. anaerobia strains, all from spoiled British beers. None of them is a type strain. When Shimwell described Achromobacter (15), later (16) Saccharomonas anaerobia, he proposed no type strain. We have been unable to obtain authentic $Z$. anaerobia cultures, isolated by the late J. L. Shimwell, either from the two breweries where he had been working or from one of his former collaborators. An authentic cotype of $Z$. anaerobia, strain D-364 (23), is no longer available (Sneath, personal communication). We are not aware of other existing strains of this species. Because of all the arguments presented in the present paper, we propose to regard $Z$. anaerobia as a synonym of $Z$. mobilis, to discontinue the use of $Z$. anaerobia as a separate species, and to include all of these strains in $Z$. mobilis subsp. mobilis. Our formal proposal agrees largely with former suggestions $(2,4,12)$ that the genus Zymomonas be represented by one species only, namely $Z$. mobilis. The proposal of Richards and Corbey (12) to relegate the species $Z$. anaerobia to variety level as $Z$. mobilis var. anaerobia is no longer acceptable, because there are no significant differences between $Z$. mobilis subsp. mobilis and $Z$. mobilis subsp. anaerobia, as explained in the present paper.

A few strains could not be included in the numerical analysis because they were received after the present study was terminated. They are $Z$. anaerobia 1 and 2 (isolated from beer in England), Z. mobilis 3 Delft from Arenga sap in Indonesia, $Z$. mobilis 6 Delft from tuak in Indonesia, and $Z$. mobilis B70 from infected British ale. According to Dadds et al. (2), strain B70 has a few features which are different from those of the stock strains NCIB 8938 and NCIB 8227 (vitamin requirements, serology, and slightly but not significantly lower $\mathrm{G}+\mathrm{C}$ content). However, not enough strains and features were included by them for comparison, and the nature of the differences they found allow no statistically meaningful taxonomic conclusions. In our experience, DNA homology (27) and/or protein electropherograms (Swings et al., J. Gen. Microbiol., in press), and a number of phenotypic features (not given here), showed that all of the above-mentioned five strains are normal members of Zymomonas mobilis subsp. mobilis.

One strain in our collection was aberrant (see Fig. 3). It was isolated in Bristol from sick cider. It was sent by B.T.P. Barker in 1951 to the Laboratory of Microbiology, Technical University, Delft, The Netherlands, as Saccharomonas pomaceae strain I. It is very likely either strain B from Barker or strain 1 of Millis (9; Millis and van der Toorn, personal communications). We received this strain from $\mathrm{Ir}$. J. van der Toorn, Delft, as $Z$. anaerobia subsp. pomaceae, the name proposed by Millis (9) for the cider sickness organism. Since $Z$. mobilis and $Z$. anaerobia are synonyms, we propose a transfer of the subspecies to $Z$. mobilis subsp. pomaceae (Millis) comb. nov. with our strain T.H. Delft (= I from Barker) as the lectotype strain. It was deposited as ATCC 29192 and NCIB 11200. 


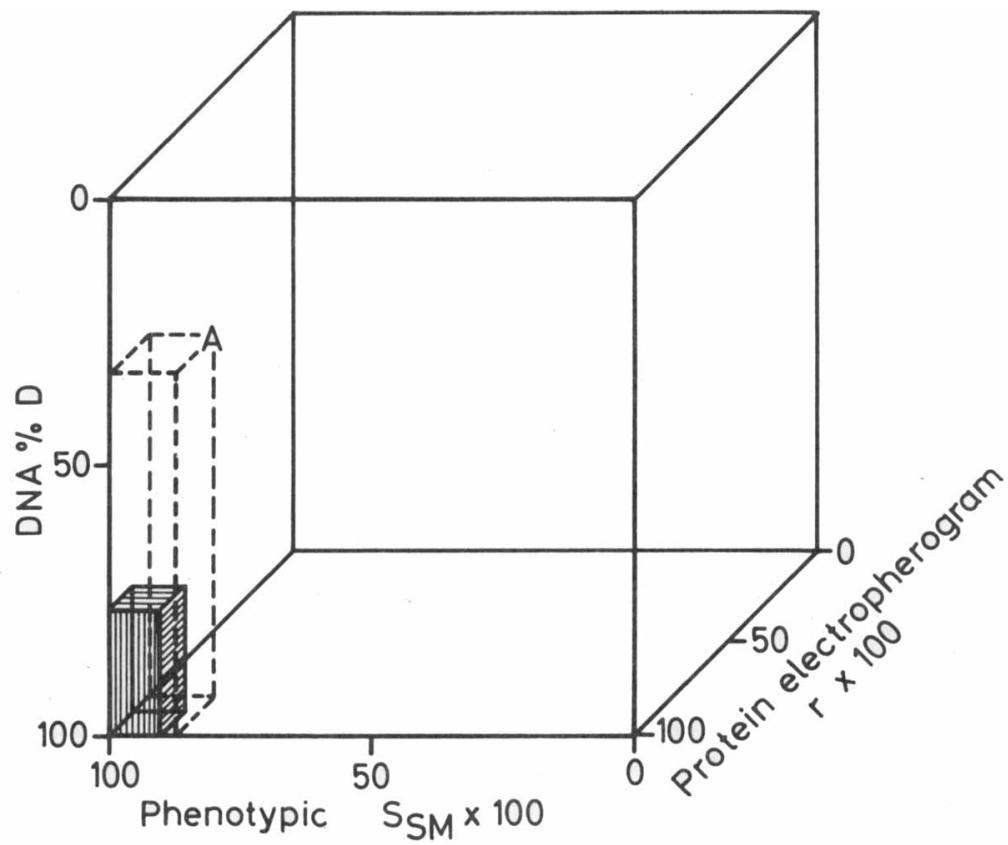

Fig. 3. Space model of the taxonomic affinities within the genus Zymomonas. The axes are: DNA relatedness, expressed in \% D; phenotypic similarity, expressed as $S_{\mathrm{SM}}$ in \%; and protein profile similarity, expressed as correlation coefficient $\times 100$. The capital A indicates the position of the type strain, ATCC 29192, of $Z$. mobilis subsp. pomaceae. All of the other strains studied are contained in the shaded lower left corner column.

Shimwell's (16) proposal to unite all nonmotile beer strains in a separate taxon was maintained by Carr (1) as $Z$. anaerobia var. immobilis (Shimwell) Carr. Our data show that motility is randomly distributed in the genus. Lindner's pulque strain was very motile when isolated and in the subsequent years; it no longer is. We observed loss of motility in two other strains. In our studies (27; Swings et al., J. Gen. Microbiol., in press; and this paper), motile and nonmotile strains did not cluster in separate groups. For all of these reasons, a separate taxon cannot be maintained for nonmotile Zymomonas strains.

- When it is accepted that $Z$. anaerobia is not needed as a separate species, $Z$. anaerobia var. anaerobia Carr (1) automatically disappears with it.

We formally propose the following classification and nomenclature of the genus Zymomonas Kluyver and van Niel 1936, 399.

Type species: Zymomonas mobilis (Lindner) Kluyver and van Niel 1936, 399.

Syn.: Termobacterium mobile Lindner 1928, 253; Pseudomonas lindneri Kluyver and Hoppenbrouwers 1931, 259; Saccharomonas lindneri (Kluyver and Hoppenbrouwers) Shim- well 1950, 182; Achromobacter anaerobium Shimwell 1937, 509; Saccharomonas anaerobia (Shimwell) Shimwell 1950, 181; Zymomonas anaerobia (Shimwell) Kluyver 1957, 199.

1. Zymomonas mobilis subsp. mobilis (Lindner) Kluyver and van Niel 1936, 399. Syn.: Zymomonas anaerobia subsp. anaerobia (Shimwell) Kluyver 1957, 199; Zymomonas anaerobia subsp. immobilis (Shimwell) Carr 1974, 353; Z . mobilis subsp. anaerobia Richards and Corbey 1974, 243. Lectotype strain: $Z$. mobilis ATCC 10988 (see above). Phenotypic centrotype: strain Z6 = ATCC $29191=$ NCIB 11199.

2. Zymomonas mobilis subsp. pomaceae (Millis) comb. nov. Syn.: Zymomonas anaerobia subsp. pomaceae Millis 1956, 527. Vernacular name: "Cider sickness organism" Barker and Hillier 1912, 78. Lectotype strain: $Z$. mobilis subsp. pomaceae T. H. Delft ex $Z$. anaerobia subsp. pomaceae (Delft) ex Saccharomonas pomaceae strain I Barker. ATCC 29192, NCIB 11200 .

\section{ACKNOWLEDGMENTS}

The senior author (J.D.L.) thanks the Fonds voor Kollektief Fundamenteel Onderzoek for research and personnel grants. We are very indebted to Ir. J. van der Toorn (Lab. Microbiologie, Technical University, Delft, The 
Netherlands) for valuable information on the origins of the type strains.

\section{REPRINT REQUESTS}

Address requests for reprints to: Prof. J. De Ley, Laboratory for Microbiology, R.U.G., K.L. Ledeganckstraat 35, B9000 Gent, Belgium.

\section{LITERATURE CITED}

1. Carr, J. G. 1974. Genus $Z$ ymomonas Kluyver and van Niel 1936, 399, p. 352-353. In R. E. Buchanan and N. E. Gibbons (ed.), Bergey's manual of determinative bacteriology, 8th ed. The Williams \& Wilkins Co., Baltimore.

2. Dadds, M. J. S., P. A. Martin, and J. G. Carr. 1973. The doubtful status of the species Zymomonas anaerobia and Z. mobilis. J. Appl. Bacteriol. 36:531-539.

3. Dawes, E. A., D. W. Ribbons, and D. A. Rees. 1966. Sucrose utilization by $Z$ ymomonas mobilis: formation of a levan. Biochem. J. 98:804-812.

4. De Ley, J. 1964. Pseudomonas and related genera. Annu. Rev. Microbiol. 18:17-46.

5. Falkow, S. 1958. Activity of lysine decarboxylase as an aid in the identification of Salmonellae and Shigellae. Am. J. Clin. Pathol. 29:598-600.

6. Kluyver, A. J., and W. J. Hoppenbrouwers. 1931. Ein merkwürdiges Gärungsbakterium: Lindner's Termobacterium mobile. Arch. Mikrobiol. 2:245-260.

7. Kovacs, N. 1956. Identification of Pseudomonas pyocyanea by the oxidase reaction. Nature (London) 178:703.

8. Lysenko, O. 1961. Pseudomonas - an attempt at a general classification. J. Gen. Microbiol. 25:379-408.

9. Millis, N. F. 1956. A study of the cider-sickness bacillus - a new variety of Zymomonas anaerobia. J. Gen. Microbiol. 15:521-528.

10. Rhodes, M. E. 1958. The cytology of Pseudomonas spp. as revealed by a silver-plating staining method. J. Gen. Microbiol. 18:639-648.

11. Rhodes, M. E. 1959. The characterization of Pseudomonas fluorescens. J. Gen. Microbiol. 21:221-263.

12. Richards, M., and D. A. Corbey. 1974. Isolation of $Z y$ momonas mobilis from primed beer. J. Inst. Brew. 80:241-244.

13. Rogers, D. J., and T. T. Tanimoto. 1960. A computer program for classifying plants. Science 132:11151118 .

14. Schreder, K., R. Brunner, and R. Hampe. 1934. Die anaerobe und aerobe Gärung von Pseudomonas lindneri-Kluyver in glucosehaltiger anorganischer Nährlösung. Biochem. Z. 273:223-242.

15. Shimwell, J. L. 1937. Study of a new type of beer disease bacterium (Achromobacter anaerobium sp. nov.) producing alcoholic fermentation of glucose. J. Inst. Brew. 43:507-509.

16. Shimwell, J. L. 1950. Saccharomonas, a proposed new genus for bacteria producing a quantitative alcoholic fermentation of glucose. J. Inst. Brew. 56:179-182.
17. Sierra, G. 1957. A simple method for the detection of lipolytic activity of micro-organisms and some observations on the influence of the contact between cells and fatty substrates. Antonie van Leeuwenhoek $J$. Microbiol. Serol. 23:15-22.

18. Silvestri, L., M. Turri, L. R. Hill, and E. Gilardi. 1962. A quantitative approach to the systematics of actinomycetes based on overall similarity, p. 333-360. In G. C. Ainsworth and P. H. A. Sneath (ed.), Microbial classification: 12th symposium of the Society for General Microbiology. Cambridge University Press, Cambridge, England.

19. Skerman, V. B. D. 1959. A guide to the identification of the genera of bacteria. The Williams \& Wilkins Co., Baltimore.

20. Sneath, P. H. A. 1957. Some thoughts on bacterial classification. J. Gen. Microbiol. 17:184-200.

21. Sneath, P. H. A., and V. G. Collins. 1974. A study in test reproducibility between laboratories: report of a Pseudomonas working party. Antonie van Jeeuwenhoek J. Microbiol. Serol. 40:481-527.

22. Sneath, P. H. A., and R. Johnson. 1972. The influence on numerical taxonomic similarities of errors in microbiological tests. J. Gen. Microbiol. 72:377-392.

23. Sneath, P. H. A., and V. B. D. Skerman. 1966. A list of type and reference strains of bacteria. Int. J. Syst. Bacteriol. 16:1-133.

24. Snell, J. J. S., and S. P. Lapage. 1973. Carbon source utilization tests as an aid to the classification of nonfermenting Gram-negative bacteria. J. Gen. Microbiol. 74:9-20.

25. Society of American Bacteriologists. 1957. Manual of microbiological methods. McGraw-Hill Book Co., New York.

26. Sokal, R. R., and C. D. Michener. 1958. A statistical method for evaluating systematic relationships. Univ. Kans. Sci. Bull. 38:1409-1438.

27. Swings, J., and J. De Ley. 1975. Genome deoxyribonucleic acid of the genus Zymomonas Kluyver and van Niel 1936: base composition, size, and similarities. Int. J. Syst. Bacteriol. 25:324-328.

28. Tanko, B. 1932. Ueber die Bildung von Acetoin durch Termobakterium mobile Lindner. Biochem. Z 247:482-485.

29. Van Pee, W., and J. Swings. 1971. Chemical and microbiological studies on congolese palm wines (Elaeis guineensis). East Afr. Agric. For. J. 36:311-314.

30. Van Pee, W., and J. Swings. 1972. Etude de quelques souches du genre Zymomonas, isolées de vins de palme zaïrois. Acad. R. Sci. Outre-Mer (Brussels). Bull. Séances p. 186-195.

31. Van Pee, W., J. Swings, and M. Vanlaar. 1974. Influence des colorants, métaux lourds et antibiotiques sur la croissance de Zymomonas. Acad. R. Sci. Outre-Mer (Brussels). Bull. Séances p. 638-645.

32. Van Pee, W., M. Vanlaar, and J. Swings. 1974. The nutrition of Zymomonas. Acad. R. Sci. Outre-Mer (Brussels). Bull. Séances p. 206-211. 